Int Arch Allergy Immunol 1995;106:431-432

\title{
Subject Index, Vol. 106, 1995
}

Acrodynia 180 Activation mutation 377 Adhesion molecule(s) 69, 345 Adult chironomid midges 271 Aging 219

Alcoholic liver cirrhosis 302 Alginate 357

Allergic bronchopulmonary aspergiUosis 263

conjunctivitis 428

contact dermatitis 166

encephalomyelitis 20

rhinitis 286 Allergy 55,118

Amino acid sequence 258 Amyloidosis 25 Anergy 3 AngiotensinI 229

- $\quad$ II 229

Antiallergic agent(s) 78,410

- $\quad$ drug 157

Antiasthmatic 78

Anticardiolipin antibody 8

Anti-CD4 activity 366

Anti-fibrillarin antibodies 180

Antigliadin antibodies 134

Anti-nucleolar antibodies 180

Antiphospholipid antibody syndrome 8

Antithrombin III 8

Apoptosis 3

Aspergilloma 263

Aspergillus 263

Aspirin-induced asthma 92

Asthma 69

-, animal model 86

-, bronchial 401

Asthmatic rhinitis, allergic 286

Atopic asthma 149

- dermatitis 55, 118

Atopy 62, 149

Autoimmunity 20, 92, 180, 213

B lymphocytes 46,55

Bacteria 97

Blast transformation 345

Blocking antibodies 250

Bone marrow 13

Bromelain-treated mouse RBC 372

Bronchial asthma 297, 410 
Candida albicans 118 CAP ${ }^{\circledR}$ system 263 Carbohydrate epitopes 146

CD28 101

Cell-mediated immunity 20

Cereal cross-reactivity 134

Chronic eosinophilic pneumonia 173

fatigue syndrome 180

granulomatous disease 425

sinusitis 416

Circulating immune complexes 366 c-kit receptor tyrosine kinase 377 Complement 305

- $\quad$ C5a 386

ConA-induced blastogenesis 219 Contact allergy 394

- $\quad$ hypersensitivity 180

Corticosteroids 20

Cre/loxP 323

Cross-reactivity 118

Cystic fibrosis 357

Cytokines 351

Cytomegalovirus 235

Delayed hypersensitivity 38 Delayed-type hypersensitivity 180 Dental amalgam 180 Dermatitis herpetiformis 134 Dermatophagoides pteronyssinus 286, 297 Diabetes mellitus 169

Dinitrofluorozene 166 Double-stranded RNA 97 DP-1904 401

EEG 97

Endothelialcell 305,335

Endothelium 69

Endotoxin 97

Eosinophil(s) 139,278, 297, 410, 428

- $\quad$ cationic protein 278

Epidemiology 149

Epithelium 69

Epstein-Barr virus 235

Experimental allergic encephalomyelitis

335 Extraocular muscles 204

Facs analysis 219 Fetal loss 8 Fever 97 Fibroblast 213 Flow cytometry 345 Fluorescenceactivated cell sorter 425 Fluorometry 241 Formaldehyde 422

Gene targeting 323

Genetics 62

Glomerulonephritis 180

Gluten-sensitive enteropathy 134

ß2-Glycoprotein I 8

Grass pollen allergen Lolp $\backslash 250$

Graves' disease 213

- $\quad$ ophthalmopathy 213

Growth inhibition 107

Guinea pig 410

Hi-receptor antagonist 78 Hapten-specific T cell 394 Heart 386

Hepatic class II antigens 219 Histamine 278,386,416 
liberation 241

release 250 HIV 366 HLA 38 HPLC 229 Human insulin 169

leukocytes 229

monoclonal antibodies 32 Hyperreactivity 86 Hyperresponsiveness 401

Idiotypes $372 \operatorname{IgE} 55,62,263,422$-, allergen-specific 46 -, allergy 175

- $\quad$ antibody 271

-, cytophotometry 241

- $\quad$ receptors 241

IgG, allergen-specific 46

IGHEgene 62

IL·2 101

$\mathrm{IL} \cdot 4$ 46, 55, 101

-, IL-13 175

receptor 46,124

-, soluble 46, $55 \mathrm{IL} \cdot 5139$

ГL·8 351,357 IL-13 175

Immediate-phase response 78 Immune complex 180

mutants 323

response 32 Immunoglobulin G2 146 Immunoglobulins 124 Immunohistochemistry 113, 345

Immunopotentiation 166 Immunoscintigraphy 113

Immunotherapy 250 Infection 97 Inflammation 305 Inflammatory mediators 416 Insulin allergy

169

- $\quad$ antibodies 169

Intercellular adhesion molecule-1 139, 302,

335 Interferon- $\gamma$ 55, $101 \alpha$-Interferon treatment 169

- $\quad$-, side effects 169

Interleukin-12 124,166

Intracellular Ca 157

In vitro immunization 32 Ionic current 157

Juvenile rheumatoid arthritis 235

Knock-in 323 Knockout 323

Late-phase response 78 Latex allergy 258

- $\quad$ proteins 258

Lectin 25

Leucocyte proliferation 20 Leukotriene C4 278,386

- C4/D4Æ4 416

Lichen planus 180

Lipopolysaccharide 335

Liver, nucleic acids 219

-, proteins 219

LolpX1 146

Low-affinity immunoglobulin E receptor

(CD23) 124 LPS activation 372 Lupus anticoagulant 8 Lymphocyte adhesion 335 Lymphocytes 410

Macrophage hybridoma 107

Mannan 118 
Mast cell(s) 241,377,386

Membrane lipid 107

Memory T cells 428

Mercury 180

Metal toxicity 180

Methotrexate 410

MHC 13

Monoclonal antibodies 113, 372

Multiple chemical sensitivities 180

- $\quad$ sclerosis 180

Muramyl peptide 97

Mycobacteria 113

Naive T cell 101 Nasal allergy 139

gland acinar cell 157

mucosa 139

reaction, early and late 278

secretions 278 Natural antibodies 305, 372 Nickel allergy 345

Non-rapid eye movement sleep 97

Occupational allergy 271 Ophthalmopathy 204 Orbital connective tissue 204 Ovalbumin 46,422

Oxazolone 166 Oxidative burst 425

Pentraxin 25

Peptidoglycan monomer 219 Phagocytes 357 Phospholipid antigens 372 Pityrosporum ovale 118

-, bronchial asthma 291 Platelet(s) 8

aggregation 291

desensitization 291 Platelet-activating factor 291 Pollinosis 149 Precipitins 263 Predictive test

394 Pregnancy 173 Prevalence 149 Prostaglandin D2 416 Protamine 386

Protein C 8

Pseudomonas aeruginosa 357

Pulmonary tuberculous lesion 113

Ragweed 46

Rantes 428

Rapid eye movement sleep 97

RAST® system 263

Rats 241

-, inbred strains 241

Receptor antagonist 175

Remaining hemoglobin 271

RIA 229

RU486 20

Rubber elongation factor 258

Saccharomyces cerevisiae 118 SAPALDIA 149 Schultz-Dale reaction 401 Seasonal allergic rhinitis 278

Sensitization 422

Sephadex-induced eosinophilia 401

Sepsis 302

Serotonin, release 291 
Serum amyloid P component 25

Sheep red blood cells 219

Single-cell measurement 425

Skin immune system 351

Specificity 32

Spinabifida 258

Spleen 219

Steroids 173

Strain difference 86

Substance P 386

Superantigen(s) 3,163

Suppressive macrophages 219

Synthetic peptides 250

Systemic lupus erythematosus 8,235

Tcell 163

- - activation 3

T-cell-mediated shock 3

Tear 428

ß-Thromboglobulin, release 291

Thrombosis 8

Thromboxane A2 401

Thymocytes 13

Thyroid 213

Thyroid-associated ophthalmopathy 204

Toxic shock syndrome 163

Trachea, guinea pig 86

Transplantation 305

Treponema pallidum antigens 32

Tryptase 278

Tuberculin 38

Tuberculosis 38

Tumor necrosis factor 163

Vascular endothelial cell 163 Vasculitis 92 Virus 97

Yeast 118

ZCR-2060 78 Zinc 219 ZnCl2 219

432

Subject Index 